\title{
Clinicopathological and prognostic features of early gastric cancer patients after surgery: a retrospective study
}

Jing Qi

Xiangya Hospital Central South University

Congbo Zhu

The First People's Hospital of Yueyang

Weihang Liu

Xiangya Hospital Central South University

Sheng Liu

Xiangya Hospital Central South University

Gaoqiang Cai

Xiangya Hospital Central South University

Guo-Qing Liao ( $\square$ liaoguoqing@medmail.com.cn )

Xiangya Hospital Central South University https://orcid.org/0000-0002-5967-0282

\section{Research}

Keywords: Early gastric cancer, prognostic factors, tumor diameter, lymph node metastasis, risk factors

Posted Date: July 24th, 2020

DOl: https://doi.org/10.21203/rs.3.rs-46111/v1

License: (1) This work is licensed under a Creative Commons Attribution 4.0 International License. Read Full License 


\section{Abstract}

Background: Despite the decline in the incidence of gastric cancer, the incidence of early gastric cancer has increased. Hence, understanding the clinicopathological and prognostic features of early gastric cancers could help us understand the development of gastric cancer and improve the prognosis of early gastric cancer.

Methods: A total of 244 patients diagnosed with early gastric cancer after surgery at Xiangya Hospital Central South University were retrospectively analyzed.

Results: General data showed that in patients with a mean age of $54.30 \pm 10.68$ years ( $M: F=1.6: 1)$, the median tumor size was $2.203 \pm 1.245 \mathrm{~cm}$. A total of $15.6 \%$ of patients had lymph node metastasis. By univariate analysis, the longest diameter of the tumor, T stage, total number of dissected lymph nodes, number of metastatic lymph nodes, metastatic-to-total dissected lymph node (LN) ratio, vascular invasion, NLRc, and MLRc were associated with disease-free survival; tumor size, invasive depths, vascular invasion, NLRc, MLRc, NWRc and LWRc were associated with lymph node metastasis.

Additionally, the longest diameter of tumor and total number of dissected lymph nodes were independent factors for early gastric cancer patients; tumor size, invasive depths, vascular invasion and NLRc were independent risk factors for lymph node metastasis in EGC.

Conclusion: The longest diameter of the tumor and total number of dissected LNs were independent prognostic factors for EGC patients. Additionally, the longest diameter of the tumor, tumor invasive depths, vascular invasion and NLRc were the independent risk factors for lymph node metastasis in EGC patients.

\section{Introduction}

In recent years, the incidence rates of gastric cancer (GC) have been declining steadily worldwide[1]. However, GC is still the fifth most common malignant tumor and the third leading cause of cancer-related mortality globally, with an estimated 1,033,701 new cases and 782,654 deaths in 2018[2, 3]. This translates into a high fatality to new case rate of $75 \%$, which is much higher than that of other prevalent solid cancers, including breast and prostate cancers[2]. The incidence of GC varies regionally, with approximately 70\% occurring in Eastern Asia, Central and Eastern Europe and South America[4]. Approximately $50 \%$ of patients are diagnosed with advanced disease, with a 5-year cancer-specific survival rate lower than $30 \%[5]$. However, the prognosis of GC markedly depends on the stage at diagnosis. In Western countries, including Europe and the United States, the 5-year survival rate does not exceed $25 \%[6]$, but in Japan, the 5 -year cancer-specific survival rate is $90 \%$ with the diagnosis of early GC of up to $50 \%[7]$.

Similar to other common cancers, GC rarely occurs in children and young persons, with a peak incidence ranging from 55 to 80 years, and gastric cancer rates are twice as high in men as in women[8]. GC is divided into early gastric cancer (EGC) and advanced gastric cancer, with significant differences in 
recurrence and cancer-related survival rates. EGC is defined as a carcinoma invading the mucosa and/or submucosa with or without lymph node metastases[9]. Despite a decline in the incidence of total gastric cancer and the aging of the population, the incidence of early gastric cancer has been steadily increasing since the late 1980 s[10].

Recent studies on EGC data remain incomplete and conflicting; with the growing incidence of early gastric cancer, it is essential to focus on the clinical and prognostic features of early gastric cancer, as they are controversial. We retrospectively examined patients with early gastric cancer in our center, aiming to achieve a better understanding of clinicopathologic features, the development of gastric cancer and prognostic factors in early gastric cancer and to improve the outcomes of gastric cancer.

\section{Patients And Methods Patient selection}

Data of patients who were diagnosed with EGC from January 2013 to December 2018 after surgery at Xiangya Hospital Central South University were collected through a pathology information system. The eligibility criteria in this study included (1) patients who were histopathologically diagnosed with early gastric cancer (carcinoma invading the mucosa and/or submucosa) after surgery; (2) no other synchronous malignant neoplasia; (3) no perioperative or postoperative chemotherapy or radiotherapy applied.

This retrospective study was supported by the Medical Ethical Committee of Xiangya Hospital, Central South University, and due to the retrospective nature of the study, informed consent was waived.

\section{Data of routine blood collection}

All patients' preoperative and postoperative peripheral routine blood tests were drawn within 7 days before the operation and 7 days after the operation. iNLR (initial preoperative neutrophil-to-lymphocyte ratio), iMLR(initial preoperative monocyte-to-lymphocyte ratio), iNWR (initial preoperative neutrophil-towhite blood cell ratio), iLWR (initial preoperative lymphocyte-to-white blood cell ratio), iPLR (initial preoperative platelet-to-lymphocyte ratio), iMWR (initial preoperative monocyte-to-white blood cell ratio), NLRc (postoperative change NLR), MLRc (postoperative change MLR), NWRc (postoperative change NWR), LWRc (postoperative change LWR), PLRc (postoperative change PLR), and MWRc (postoperative change NWR) were determined by ROC curve analysis.

\section{Follow-up data}

Patients who underwent surgery were followed every 3 months for the first 2 years, every 6 months between 2 and 5 years, and then every 12 months thereafter. We supplemented the follow-ups with our outpatient system or communicated with patients by telephone. We examined the postoperative EGC patients by chest scan, abdominal CT scan, magnetic resonance imaging (MRI) scan, abdominal ultrasound scan, endoscopy (including gastroscopy and colonoscopy) and positron emission 
tomography-computerized tomography (PET-CT) (if necessary) to evaluate tumor recurrence and distant metastasis. All patients were monitored until October 1, 2019, or death. Metastatic recurrence revealed by imaging and death were regarded as the endpoint events.

\section{X-tile and statistical analyses}

Patient characteristics between each group were compared by chi-square test. The values for the iNLR, iMLR, iPLR, iNWR, iLWR, iMWR, NLRc, MLRc, PLRc, NWRc, LWRc, MWRc, longest diameter of the tumor, total number of dissected lymph nodes (LNs), number of metastatic LNs, and ratio of metastatic-to-total dissected LNs were determined by receiver operating characteristic (ROC) curve, based on the results of the ROC curve, X-tile analyses were conducted to assess the optimal cutoff values based on the integral optic density (IOD). The disease-free survival (DFS) and overall survival (OS) were calculated by the Kaplan-Meier method, and differences between Kaplan-Meier curves were investigated by the log-rank test. Significant predictors for survival identified by univariate analysis were further assessed by multivariate analysis using a multivariate Cox proportional hazards regression model (forward stepwise method, conditional likelihood ratio). The significant predictors for lymph node metastasis by univariate analysis were further assessed by logistic regression. Statistical analyses were performed using SPSS V22.0 (SPSS Inc., USA).P value was considered statistically significant below the $5 \%$ level.

\section{Results}

\section{Clinicopathologic features for EGC patients}

For our research, 244 patients were eventually enrolled as our study group (Fig. 1). As shown in Table 1, the EGC group comprised 149 males and 95 females, and the male-to-female ratio was approximately $1.6: 1$, with a mean age of $54.30 \pm 10.68$ years, ranging from 24 to 82 years. All patients underwent D2 lymphadenectomy and curative gastrectomy (68.9\% underwent the open method and $31.3 \%$ underwent the laparoscopy method). Most EGC patients underwent Billroth II reconstruction (71.7\%), the tumor was most commonly located in the antrum and pylorus (61.9\%), and the average size of the longest diameter of the tumor was $22.03 \pm 12.45 \mathrm{~mm}$, ranging from 3 to $85 \mathrm{~mm}$. The most common pathological features included moderate-poor differentiation (58.2\%), T1b (50.4\%), N0 (84.4\%), non-perineurium invasion invasion(99.2\%), nonvascular invasion (90.6\%), HER2-negative immunohistochemistry $(81.6 \%)$ and TMN stage la (76.6\%). More details are shown in Table 1. 
Table 1

Characterization of the study group

\begin{tabular}{|c|c|c|}
\hline Quality & & $\mathrm{N}(\%)$ or mean $\pm \mathrm{SD}$ \\
\hline Mean Age[years](range) & & $54.30 \pm 10.68(24-82)$ \\
\hline \multirow[t]{2}{*}{ Gender } & Male & $149(61.1 \%)$ \\
\hline & Female & $95(38.9 \%)$ \\
\hline \multirow[t]{2}{*}{ Methods for operation } & Open method & $168(68.9 \%)$ \\
\hline & Laparoscopic method & $76(31.3 \%)$ \\
\hline \multirow[t]{3}{*}{ Methods for reconstruction } & Billroth I & $36(14.8 \%)$ \\
\hline & Billroth II & $175(71.7 \%)$ \\
\hline & Roux-en-Y & $33(13.5 \%)$ \\
\hline \multirow[t]{4}{*}{ Localization } & Cardia & $6(2.5 \%)$ \\
\hline & Fundus & $21(8.6 \%)$ \\
\hline & Body of stomach & $66(27.0 \%)$ \\
\hline & Antrum and pylorus & $151(61.9 \%)$ \\
\hline Tumor maximal dimension [mm] (range) & & $22.03 \pm 12.45(3-85)$ \\
\hline \multirow[t]{2}{*}{ Tumor differentiation } & Well & $102(41.8 \%)$ \\
\hline & Moderate-poor & $142(58.2 \%)$ \\
\hline \multirow[t]{3}{*}{ T stage } & Tis & $17(7.0 \%)$ \\
\hline & T1a & $104(42.6 \%)$ \\
\hline & $\mathrm{T} 1 \mathrm{~b}$ & $123(50.4 \%)$ \\
\hline \multirow[t]{4}{*}{ N stage } & NO & $206(84.4 \%)$ \\
\hline & N1 & $23(9.4 \%)$ \\
\hline & N2 & $11(4.5 \%)$ \\
\hline & N3a & $4(1.6 \%)$ \\
\hline Numbers of lymph nodes dissection & & $20.92 \pm 7.53(4-51)$ \\
\hline Metastatic numbers of lymph nodes dissection & & $0.45 \pm 1.44(0-10)$ \\
\hline \multirow[t]{2}{*}{ Perineuronal invasion } & Yes & $2(0.08 \%)$ \\
\hline & No & $242(99.2 \%)$ \\
\hline vascular invasion & Yes & $23(9.4 \%)$ \\
\hline
\end{tabular}




\begin{tabular}{|lll|}
\hline Quality & & N(\%) or mean \pm SD \\
\hline Immunohistochemistry score for her-2 & 0 & $221(90.6 \%)$ \\
& + & $199(81.6 \%)$ \\
\hline TNM stage* & ++ & $26(10.7 \%)$ \\
& +++ & $14(5.7 \%)$ \\
& 0 & $5(2.0 \%)$ \\
\hline la & $17(7.0 \%)$ \\
\hline Ib & $187(76.6 \%)$ \\
\hline *according to the NCCN guidelines Gastric cancer(2019,Version 4) & $25(10.2 \%)$ \\
\hline
\end{tabular}

\section{$\mathrm{ROC}$ analysis and $\mathrm{X}$-tile analyses}

The values of iNLR, NLRc, iMLR, MLRc, iPLR, PLRc, iNWR, NWRc, iLWR LWRc, iMWR and MWRc were calculated by using ROC curves. Results showed the areas under the ROC curve for NLRc and MLRc were 0.632 (95\% Cl: 0.519-0.744; $\mathrm{P}=0.046)$ and 0.659 (95\% Cl: 0.549-0.768; $\mathrm{P}=0.016$ ), respectively (Table 2, Fig. 2a-e). Besides, NLRc $=7.80$ and MLRc $=1.40$ were the optimal cutoff points determined by the X-tile program (Fig. 3a-b). 
Table 2

Area under the curve(AUC) identified by Disease-free Survival

\begin{tabular}{|c|c|c|c|}
\hline Variable & Area & $P$ value & $95 \% \mathrm{Cl}$ \\
\hline iNLR & 0.510 & 0.882 & $0.380-0.639$ \\
\hline iMLR & 0.543 & 0.516 & $0.420-0.666$ \\
\hline iPLR & 0.571 & 0.281 & $0.448-0.695$ \\
\hline iNWR & 0.512 & 0.859 & $0.381-0.643$ \\
\hline iLWR & 0.484 & 0.806 & $0.359-0.609$ \\
\hline iMWR & 0.508 & 0.898 & $0.369-0.648$ \\
\hline NLRc & 0.632 & 0.046 & $0.519-0.744$ \\
\hline MLRc & 0.659 & 0.016 & $0.549-0.768$ \\
\hline PLRc & 0.536 & 0.589 & $0.399-0.672$ \\
\hline NWRc & 0.566 & 0.315 & $0.441-0.692$ \\
\hline LWRc & 0.611 & 0.085 & $0.489-0.734$ \\
\hline MWRc & 0.524 & 0.714 & $0.397-0.652$ \\
\hline Tumor maximal dimension & 0.712 & 0.001 & $0.617-0.808$ \\
\hline Total LN dissection numbers & 0.690 & 0.003 & $0.573-0.807$ \\
\hline Metastatic LN dissection numbers & 0.764 & $\mathbb{\nabla 0 . 0 0 1}$ & $0.634-0.894$ \\
\hline Metastatic-to-total LN dissection numbers ratio & 0.770 & 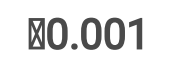 & $0.639-0.901$ \\
\hline
\end{tabular}

In addition, results also indicated that the areas under the ROC curve for the longest diameter of the tumor, total number of dissected $\mathrm{LNs}$, number of metastatic $\mathrm{LNs}$ and ratio of metastatic-to-total dissected LNs were 0.712 (95\% Cl: 0.617-0.808; $\mathrm{P}=0.001), 0.690$ (95\% Cl: $0.573-0.807 ; \mathrm{P}=0.003), 0.764$ (95\% Cl: $0.634-0.894 ; \mathrm{P} \otimes 0.001$ ), and 0.770 (95\% Cl: 0.639-0.901; P囚0.001), respectively (Table 2, Fig. 2f-i). Moreover, the best cutoff values were $2.0 \mathrm{~cm}$ for the diameter of the tumor, 13 for the total number of dissected LNs, 1 for the number of metastatic LNs, and $6 \%$ for the ratio of metastatic-to-total dissected LNs determined by X-tile program (Fig. 3c-f).

\section{Risk factors for lymph node(LN) metastasis in EGC patient}

Since the $L N$ metastasis plays a critical role for the selection of clinical treatment (EMR/ESD or surgery) in EGC patients, it is essential to identify the risk factors for $L N$ metastasis in EGC patients. ROC curve 
was conducted to calculate the best cutoff points of each viriate for the $L N$ metastasis. Results showed that the optimal cutoff points of NLRc, MLRc, NWRc, LWRc, and tumor maximal dimension were 5.29, $0.49,0.70,0.13$ and 1.9 , respectively(Table S1, Figure S1). Next, we investigated the risk factors for lymph node metastasis according to univariate analysis. As shown in Table 3, tumor maximal dimension, $\mathrm{T}$ stage, vascular invasion, NLRc, MLRc, NWRc, and LWRc were associated with LN metastasis in EGC patients. Moreover, through multivariate analysis we found that tumor maximal dimension $(\leq 1.9$ / $\otimes 1.9 \mathrm{~cm})(\mathrm{RR}: 2.421, \mathrm{p}=0.044)$, $\mathrm{T}$ stage (Tis + T1a/T1b) (RR:3.807, $\mathrm{p}=0.004)$, vascular invasion (yes/no) $(R R: 0.241, p=0.006)$ and NLRc $(\leq 5.29 / \varangle 5.29)(R R: 8.500, p=0.010)$ were the independent risk factors for LN metastasis in EGC patients (Table 4). 
Table 3

Risk Factors for LN metastasis in EGC patients according to univariate analysis

\begin{tabular}{|c|c|c|c|c|c|}
\hline Risk factors & & LN- & LN+ & $95 \% \mathrm{Cl}$ & $P$ value \\
\hline \multirow[t]{2}{*}{ Age(years) } & $\nabla 60$ & 129 & 28 & $0.234-1.156$ & 0.104 \\
\hline & $\geq 60$ & 77 & 10 & & \\
\hline \multirow[t]{2}{*}{ Gender } & Male & 129 & 20 & $0.751-3.026$ & 0.246 \\
\hline & Female & 77 & 18 & & \\
\hline \multirow[t]{2}{*}{ Methods for operation } & Open method & 141 & 27 & $0.413-1.890$ & 0.750 \\
\hline & Laparoscopic method & 65 & 11 & & \\
\hline \multirow[t]{3}{*}{ Methods for reconstruction } & Billroth I & 33 & 3 & - & 0.310 \\
\hline & Billroth II & 144 & 31 & & \\
\hline & Roux-en-Y & 29 & 4 & & \\
\hline \multirow[t]{4}{*}{ Localization } & Cardia & 5 & 1 & - & 0.752 \\
\hline & Fundus & 18 & 3 & & \\
\hline & Body of stomach & 53 & 13 & & \\
\hline & Antrum and pylorus & 130 & 21 & & \\
\hline \multirow{2}{*}{$\begin{array}{l}\text { Tumor maximal dimension } \\
\text { [cm] }\end{array}$} & $\leq 1.9$ & 93 & 9 & $1.196-5.882$ & 0.014 \\
\hline & $\bigotimes 1.9$ & 113 & 29 & & \\
\hline \multirow[t]{2}{*}{ Tumor differentiation } & Well & 91 & 12 & $0.820-3.583$ & 0.149 \\
\hline & Moderate-poor & 115 & 26 & & \\
\hline \multirow[t]{3}{*}{ T stage } & Tis & 17 & 0 & - & 0.005 \\
\hline & T1a & 94 & 10 & & \\
\hline & $\mathrm{T} 1 \mathrm{~b}$ & 95 & 28 & & \\
\hline \multirow[t]{2}{*}{ Perineuronal invasion } & Yes & 1 & 1 & $0.011-2.950$ & 0.178 \\
\hline & No & 205 & 37 & & \\
\hline \multirow[t]{2}{*}{ vascular invasion } & Yes & 11 & 12 & $0.049-0.305$ & $凶 0.001$ \\
\hline & No & 195 & 26 & & \\
\hline \multirow{3}{*}{$\begin{array}{l}\text { Immunohistochemistry score } \\
\text { (Her-2) }\end{array}$} & 0 & 171 & 28 & - & 0.119 \\
\hline & + & 18 & 8 & & \\
\hline & ++ & 12 & 2 & & \\
\hline
\end{tabular}




\begin{tabular}{|c|c|c|c|c|c|}
\hline \multirow[t]{2}{*}{ Risk factors } & & LN- & LN+ & $95 \% \mathrm{Cl}$ & $P$ value \\
\hline & +++ & 5 & 0 & & \\
\hline \multirow[t]{2}{*}{ NLRc } & $\leq 5.29$ & 93 & 7 & $1.535-8.655$ & 0.002 \\
\hline & $\triangle 5.29$ & 113 & 31 & & \\
\hline \multirow[t]{2}{*}{ MLRc } & $\leq 0.49$ & 50 & 2 & $1.341-24.819$ & 0.009 \\
\hline & $凶 0.49$ & 20 & 5 & & \\
\hline \multirow[t]{2}{*}{ NWRc } & $\leq 0.70$ & 70 & 5 & $1.270-9.086$ & 0.011 \\
\hline & $凶 0.70$ & 136 & 33 & & \\
\hline \multirow[t]{2}{*}{ NLRc } & $\leq 0.13$ & 106 & 27 & $0.203-0.916$ & 0.026 \\
\hline & $\varangle 0.13$ & 100 & 11 & & \\
\hline \multicolumn{6}{|c|}{$\begin{array}{l}\text { LN: lymph node; EGC: early gastric cancer; EGC: early gastric cancer; cm: centimetre; LN: lymph node; } \\
\text { NLRc: postoperative neutrophil-to-lymphocyte ratio change; MLRC: postoperative monocyte-to- } \\
\text { lymphocyte ratio change; NWRC: postoperative neutrophil-to-white blood cell ratio change; LWRc: } \\
\text { postoperative lymphocyte -to-white blood cell ratio change. }\end{array}$} \\
\hline
\end{tabular}

Table 4

Risk Factors for LN metastasis in EGC patients according to multivariate analysis

\begin{tabular}{|c|c|c|c|c|c|}
\hline Risk factors & B & SE & RR & $95 \% \mathrm{Cl}$ & $P$ value \\
\hline Tumor maximal dimension $(\leq 1.9 / \otimes 1.9 \mathrm{~cm})$ & 0.884 & 0.440 & 2.421 & $1.023-5.799$ & 0.044 \\
\hline T stage(Tis + T1a/T1b) & 1.337 & 0.464 & 3.807 & $1.533-9.452$ & 0.004 \\
\hline vascular invasion(yes/no) & -1.423 & 0.515 & 0.241 & $0.088-0.661$ & 0.006 \\
\hline $\operatorname{NLRc}(\leq 5.29 /$ / 5.29$)$ & 2.140 & 0.826 & 8.500 & $1.683-42.914$ & 0.010 \\
\hline $\operatorname{MLRc}(\leq 0.49 / \otimes 0.49)$ & 1.178 & 0.828 & 3.246 & $0.642-16.453$ & 0.154 \\
\hline $\operatorname{MLRc}(\leq 0.70 / \otimes 0.70)$ & 0.286 & 0.661 & 1.331 & $0.364-4.865$ & 0.665 \\
\hline $\operatorname{MLRc}(\leq 0.13 / \varangle 0.13)$ & 1.347 & 0.699 & 3.844 & $0.978-15.117$ & 0.054 \\
\hline \multicolumn{6}{|c|}{$\begin{array}{l}\text { LN: lymph node; EGC: early gastric cancer; EGC: early gastric cancer; cm: centimetre; LN: lymph node; } \\
\text { NLRC: postoperative neutrophil-to-lymphocyte ratio change; MLRC: postoperative monocyte-to- } \\
\text { lymphocyte ratio change; NWRc: postoperative neutrophil-to-white blood cell ratio change; LWRC: } \\
\text { postoperative lymphocyte -to-white blood cell ratio change. }\end{array}$} \\
\hline
\end{tabular}

\section{Prognostic features for EGC}

We further investigated the relationship between the clinicopathologic features and prognosis of EGC patients according to univariate analysis. As shown in Table 5, we found the longest diameter of the tumor $>2 \mathrm{~cm}, T 1 \mathrm{~b}$, total number of dissected LNs $\leq 13$, number of metastatic $L N s \geq 1$, ratio of metastaticto-total dissected $L N s \geq 6 \%$, vascular invasion, NLRc $>7.80, M L R c>1.40$ were both associated with 
recurrence/metastasis and overall survival in EGC patients. Next, Cox proportional hazard models were performed to identify independent prognostic factors. Those identified as significant prognostic factors by univariate analysis were further assessed by a multivariate analysis. The multivariate analysis results

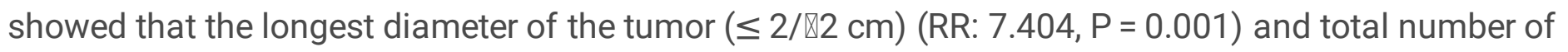
dissected LNs $(\leq 13 />13)$ (RR: $0.289, P=0.015)$ were independent risk factors for recurrence/metastasis

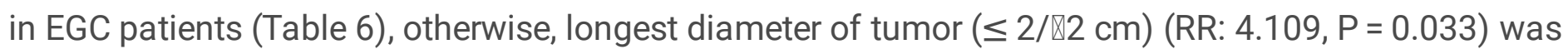
the independent risk factors for overall survival in EGC patients(Table 7). The DFS and OS for the significant prognostic factors identified by the multivariate analysis is presented in Fig. 5 . 
Table 5

Prognostic Factors in EGC patients according to univariate analysis

\begin{tabular}{|c|c|c|c|c|c|c|}
\hline \multirow[t]{2}{*}{ Prognostic factors } & & \multirow[t]{2}{*}{ Number } & \multicolumn{2}{|l|}{ DFS } & \multicolumn{2}{|l|}{ os } \\
\hline & & & $95 \% \mathrm{Cl}$ & $\begin{array}{l}P \\
\text { value }\end{array}$ & $95 \% \mathrm{Cl}$ & $\begin{array}{l}P \\
\text { value }\end{array}$ \\
\hline \multirow[t]{2}{*}{ Age(years) } & $\$ 60$ & 158 & $\begin{array}{l}72.311- \\
78.587\end{array}$ & 0.140 & $\begin{array}{l}72.744- \\
78.874\end{array}$ & 0.941 \\
\hline & $\geq 60$ & 86 & $\begin{array}{l}64.002- \\
74.879\end{array}$ & & $\begin{array}{l}70.292- \\
78.258\end{array}$ & \\
\hline \multirow[t]{2}{*}{ Gender } & Male & 149 & $\begin{array}{l}69.120- \\
75.992\end{array}$ & 0.700 & $\begin{array}{l}72.373- \\
77.898\end{array}$ & 0.263 \\
\hline & Female & 95 & $\begin{array}{l}69.057- \\
78.181\end{array}$ & & $\begin{array}{l}69.625- \\
78.659\end{array}$ & \\
\hline \multirow{2}{*}{$\begin{array}{l}\text { Methods for } \\
\text { operation }\end{array}$} & Open method & 168 & $\begin{array}{l}69.457- \\
75.631\end{array}$ & 0.738 & $\begin{array}{l}72.109- \\
77.252\end{array}$ & 0.424 \\
\hline & $\begin{array}{l}\text { Laparoscopic } \\
\text { method }\end{array}$ & 76 & $\begin{array}{l}65.962- \\
78.996\end{array}$ & & $\begin{array}{l}67.532- \\
79.906\end{array}$ & \\
\hline \multirow{3}{*}{$\begin{array}{l}\text { Methods for } \\
\text { reconstruction }\end{array}$} & Billroth I & 36 & $\begin{array}{l}74.225- \\
80.517\end{array}$ & 0.259 & $\begin{array}{l}74.423- \\
80.452\end{array}$ & 0.177 \\
\hline & Billroth II & 175 & $\begin{array}{l}68.084- \\
75.918\end{array}$ & & $\begin{array}{l}70.645- \\
77.748\end{array}$ & \\
\hline & Roux-en-Y & 33 & $\begin{array}{l}62.928- \\
77.358\end{array}$ & & $\begin{array}{l}69.810- \\
77.904\end{array}$ & \\
\hline \multirow[t]{4}{*}{ Localization } & Cardia & 6 & - & 0.583 & - & 0.909 \\
\hline & Fundus & 21 & - & & - & \\
\hline & $\begin{array}{l}\text { Body of } \\
\text { stomach }\end{array}$ & 66 & - & & - & \\
\hline & $\begin{array}{l}\text { Antrum and } \\
\text { pylorus }\end{array}$ & 151 & - & & - & \\
\hline \multirow{2}{*}{$\begin{array}{l}\text { Tumor maximal } \\
\text { dimension[cm] }\end{array}$} & $\leq 2$ & 140 & $\begin{array}{l}75.813- \\
80.586\end{array}$ & $凶 0.001$ & $\begin{array}{l}76.621- \\
80.909\end{array}$ & 0.004 \\
\hline & $\llbracket 2$ & 104 & $\begin{array}{l}60.953- \\
71.988\end{array}$ & & $\begin{array}{l}65.094- \\
74.938\end{array}$ & \\
\hline \multirow[t]{2}{*}{ Tumor differentiation } & Well & 103 & $\begin{array}{l}\text { 68.198- } \\
76.818\end{array}$ & 0.964 & $\begin{array}{l}70.182- \\
77.897\end{array}$ & 0.809 \\
\hline & Moderate-poor & 141 & $\begin{array}{l}70.358- \\
77.545\end{array}$ & & $\begin{array}{l}72.973- \\
79.132\end{array}$ & \\
\hline
\end{tabular}




\begin{tabular}{|c|c|c|c|c|c|c|}
\hline \multirow[t]{2}{*}{ Prognostic factors } & & \multirow[t]{2}{*}{ Number } & \multicolumn{2}{|l|}{ DFS } & \multicolumn{2}{|l|}{ OS } \\
\hline & & & $95 \% \mathrm{Cl}$ & $\begin{array}{l}P \\
\text { value }\end{array}$ & $95 \% \mathrm{Cl}$ & $\begin{array}{l}P \\
\text { value }\end{array}$ \\
\hline \multirow[t]{2}{*}{ T stage } & Tis + T1a & 121 & $\begin{array}{l}72.946- \\
78.524\end{array}$ & 0.013 & $\begin{array}{l}75.091- \\
79.215\end{array}$ & 0.019 \\
\hline & T1b & 123 & $\begin{array}{l}\text { 65.677- } \\
75.136\end{array}$ & & $\begin{array}{l}68.529- \\
77.093\end{array}$ & \\
\hline \multirow[t]{2}{*}{ Total LN numbers } & $\leq 13$ & 32 & $\begin{array}{l}56.160- \\
74.715\end{array}$ & 0.006 & $\begin{array}{l}61.206- \\
77.822\end{array}$ & 0.017 \\
\hline & ه13 & 212 & $\begin{array}{l}71.386- \\
76.631\end{array}$ & & $\begin{array}{l}\text { 73.116- } \\
77.687\end{array}$ & \\
\hline \multirow{2}{*}{$\begin{array}{l}\text { Metastastic } \\
\text { LN numers }\end{array}$} & 0 & 206 & $\begin{array}{l}\text { 41.107- } \\
56.570\end{array}$ & $凶 0.001$ & $\begin{array}{l}77.240- \\
80.725\end{array}$ & $凶 0.001$ \\
\hline & $\geq 1$ & 38 & $\begin{array}{l}39.656- \\
49.126\end{array}$ & & $\begin{array}{l}46.925- \\
63.573\end{array}$ & \\
\hline \multirow{2}{*}{$\begin{array}{l}\text { Metastastic-to-total } \\
\text { LN ratio }\end{array}$} & $\llbracket 6 \%$ & 219 & $\begin{array}{l}\text { 75.462- } \\
79.773\end{array}$ & $\llbracket 0.001$ & $\begin{array}{l}\text { 77.487- } \\
80.744\end{array}$ & $凶 0.001$ \\
\hline & $\geq 6 \%$ & 25 & $\begin{array}{l}32.105- \\
50.481\end{array}$ & & $\begin{array}{l}37.059- \\
57.319\end{array}$ & \\
\hline \multirow{2}{*}{$\begin{array}{l}\text { Perineuronal } \\
\text { invasion }\end{array}$} & Yes & 2 & - & 0.656 & - & 0.682 \\
\hline & No & 242 & - & & - & \\
\hline \multirow{2}{*}{$\begin{array}{l}\text { vascular } \\
\text { invasion }\end{array}$} & Yes & 23 & $\begin{array}{l}33.024- \\
54.850\end{array}$ & $凶 0.001$ & $\begin{array}{l}35.285- \\
54.730\end{array}$ & $凶 0.001$ \\
\hline & No & 221 & $\begin{array}{l}\text { 73.715- } \\
78.722\end{array}$ & & $\begin{array}{l}76.566- \\
80.299\end{array}$ & \\
\hline \multirow{4}{*}{$\begin{array}{l}\text { Immunohistochemistry } \\
\text { score for Her-2 }\end{array}$} & 0 & 199 & - & 0.410 & - & 0.369 \\
\hline & + & 26 & - & & - & \\
\hline & ++ & 14 & - & & - & \\
\hline & +++ & 5 & - & & - & \\
\hline \multirow[t]{2}{*}{ NLRc } & $\leq 7.80$ & 149 & $\begin{array}{l}74.102- \\
79.649\end{array}$ & 0.014 & $\begin{array}{l}75.453- \\
80.315\end{array}$ & 0.045 \\
\hline & ه7.80 & 95 & $\begin{array}{l}60.067- \\
71.073\end{array}$ & & $\begin{array}{l}64.168- \\
73.808\end{array}$ & \\
\hline \multirow[t]{2}{*}{ MLRc } & $\leq 1.40$ & 219 & $\begin{array}{l}\text { 72.776- } \\
78.148\end{array}$ & 0.002 & $\begin{array}{l}\text { 74.550- } \\
79.242\end{array}$ & 0.014 \\
\hline & $\bigotimes 1.40$ & 25 & $\begin{array}{l}43.446- \\
64.243\end{array}$ & & $\begin{array}{l}49.086- \\
68.157\end{array}$ & \\
\hline
\end{tabular}




\begin{tabular}{|c|c|c|c|c|c|}
\hline \multirow[t]{2}{*}{ Prognostic factors } & \multirow[t]{2}{*}{ Number } & \multicolumn{2}{|l|}{ DFS } & \multicolumn{2}{|l|}{ OS } \\
\hline & & $95 \% \mathrm{Cl}$ & $\begin{array}{l}P \\
\text { value }\end{array}$ & $95 \% \mathrm{Cl}$ & $\begin{array}{l}P \\
\text { value }\end{array}$ \\
\hline
\end{tabular}

Table 6

Prognostic Factors for DFS in EGC patients according to multivariate analysis

\begin{tabular}{|c|c|c|c|c|c|}
\hline Prognostic factors & B & SE & RR & $95 \% \mathrm{Cl}$ & $P$ value \\
\hline 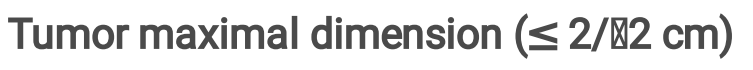 & 2.002 & 0.604 & 7.404 & $2.265-24.196$ & 0.001 \\
\hline T stage(Tis + T1a/T1b) & 0.825 & 0.568 & 2.281 & $0.750-6.939$ & 0.146 \\
\hline Total LN numbers ( $\leq 13 / 囚 13)$ & -1.242 & 0.513 & 0.289 & $0.106-0.789$ & 0.015 \\
\hline Metastastic $L N$ numers $(0 / \geq 1)$ & 0.965 & 1.157 & 2.626 & $0.272-25.336$ & 0.404 \\
\hline Metastastic-to-total LN ratio( $\leq 6 \% / \otimes 6 \%)$ & 0.970 & 1.149 & 2.637 & $0.277-25.077$ & 0.399 \\
\hline vascular invasion(yes/no) & -0.517 & 0.571 & 0.596 & $0.195-1.824$ & 0.365 \\
\hline $\operatorname{NLRc}(\leq 7.80 / \otimes 7.80)$ & 0.764 & 0.544 & 2.148 & $0.740-6.235$ & 0.160 \\
\hline $\operatorname{MLRc}(\leq 1.40 / \otimes 1.40)$ & 0.872 & 0.564 & 2.392 & $0.792-7.228$ & 0.122 \\
\hline
\end{tabular}


Table 7

Prognostic Factors for OS in EGC patients according to multivariate analysis

\begin{tabular}{|c|c|c|c|c|c|}
\hline Prognostic factors & B & SE & RR & $95 \% \mathrm{Cl}$ & $\begin{array}{l}P \\
\text { value }\end{array}$ \\
\hline 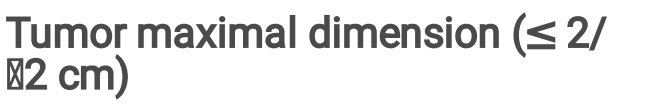 & 1.413 & 0.665 & 4.109 & $1.117-15.121$ & 0.033 \\
\hline T stage(Tis + T1a/T1b) & 0.601 & 0.738 & 1.825 & $0.429-7.759$ & 0.415 \\
\hline Total LN numbers ( $\leq 13 / 囚 13)$ & -0.952 & 0.606 & 0.386 & $0.118-1.266$ & 0.116 \\
\hline Metastastic $L N$ numers $(0 / \geq 1)$ & -7.753 & 79.179 & 0.000 & $\begin{array}{l}0.000-1.072 \mathrm{E}+ \\
64\end{array}$ & 0.922 \\
\hline $\begin{array}{l}\text { Metastastic-to-total LN ratio( } \leq 6 \% / \\
\forall 6 \%)\end{array}$ & 9.569 & 79.179 & 14315.765 & $\begin{array}{l}0.000-3.570 \mathrm{E}+ \\
71\end{array}$ & 0.904 \\
\hline vascular invasion(yes/no) & -1.090 & 0.703 & 0.336 & $0.085-1.333$ & 0.121 \\
\hline $\operatorname{NLRc}(\leq 7.80 / \otimes 7.80)$ & 0.413 & 0.653 & 1.511 & $0.421-5.428$ & 0.527 \\
\hline $\operatorname{MLRc}(\leq 1.40 / \otimes 1.40)$ & 0.781 & 0.713 & 2.183 & $0.540-8.829$ & 0.274 \\
\hline \multicolumn{6}{|c|}{$\begin{array}{l}\text { OS: overall survival, EGC: early gastric cancer, Cl: confidence interval, RR: relative risk, LN: lymph node } \\
\mathrm{cm} \text { : centimetre, NLRc = postoperative neutrophil-to-lymphocyte ratio change, MLRc = postoperative } \\
\text { monocyte-to-lymphocyte ratio change. }\end{array}$} \\
\hline
\end{tabular}

\section{Discussion}

Gastric cancer (GC) has been a critical health burden due to its high morbidity and mortality worldwide, with approximately 1,033,701 new cases and 782,654 deaths in 2018[2]. Gastric cancer can be divided into early gastric cancer (EGC) and advanced gastric cancer, with significant differences in prognosis. Moreover, despite the declining incidence of $\mathrm{GC}$ and due to the advancement of early diagnostic techniques and health examinations, the incidence of EGC seems to have steadily increased since the late 1980s[10-11]. Many studies have indicated clinicopathologic features, multiple genes and molecules in advanced gastric cancer; however, there are limited studies focusing on the clinicopathologic and prognostic features of EGC.

Our retrospective analysis of 244 patients with EGC found that the male-to-female ratio was 1.6:1, indicated that gastric cancer is a sex-related carcinoma regardless of whether early gastric cancer or overall gastric cancer patients were included, similar results were indicated by McGuire, $S$ [8]. We further investigated the prognostic features in EGC and found no significant differences in age, sex, surgical methods, reconstruction methods, tumor location, tumor differentiation, perineurium invasion or immunohistochemistry score for HER2. As our previous study indicated that laparoscopic distal gastrectomy achieves the same degree of radicality and short-term prognosis as open distal gastrectomy[12], we now verified no significant difference between laparoscopic and open surgical methods in terms of long-term survival of GC patients. Additionally, we found that HER2 expression had 
no prognostic influence in EGC. However, some retrospective studies have suggested that HER2 positivity is the second worst prognostic factor $[13,14]$, whereas other studies have suggested that HER2 status has no relationship with short- and long-term survival according to univariate and multivariate analyses[15].

We found that the longest diameter of the tumor $=2 \mathrm{~cm}$ were the best cutoff points for the prognosis factor in our large-scale group of EGC patients. Furthermore, the longest diameter of the tumor $(\leq 2 /$ $\nabla 2 \mathrm{~cm})$ and total number of dissected $\mathrm{LNs}(\leq 13 / \nabla 13)$ were independent risk factors for EGC patients according to multivariate analysis, with RRs of $7.404(P=0.001)$ and $0.289(P=0.015)$, respectively. Li $Y$ reported that lesions of over $2 \mathrm{~cm}$ might be more likely to have lymph node metastasis in EGC[17]. Similar results have been verified by $\mathrm{Li} \mathrm{H}$ et al.[18]. Our research is the first to identify the relationship between the longest diameter of the tumor and disease-free survival rate by using X-tile software and ROC curves.

The radical dissection of lymph nodes is a highly effective procedure in gastric cancer to improve the prognosis of GC and limit LN metastasis. Our study found that LN metastasis was $13.9 \%$ in EGC, which is higher than the LN metastasis rate of 2.5-8.6\% in Japan[19]. In addition, NCCN guidelines indicated that the number of dissected LNs in GC should be greater than 15; due to the lower incidence of LN metastasis in EGC, lymphadenectomy is always applied with D1+, modified D2 or D2. However, Wu H et al, indicated that in EGC patients with unknown LN status, D2 dissection was the first choice, which could prolong the survival time for those patients[20]; a similar suggestion was given by Korean and Japanese investigators[21, 22]. Our research also showed that the number of dissected LNs lower than 13 was associated with a poor prognosis of EGC patients, and this might be because limited LN dissection might lead to residual cancer, increasing the risk of recurrence or metastasis.

Since lymph node metastasis remains a critical role in the therapeutic approach (EMR/ESD vs surgery) for EGC patients, it is important to identify the risk factors for EGC patients[23]. Our study demonstrated that longest diameter of the tumor $(\leq 1.9 / \otimes 1.9 \mathrm{~cm})$, $T$ stage ( $\mathrm{T}$ is $+\mathrm{T} 1 \mathrm{a} / \mathrm{T} 1 \mathrm{~b})$, vascular invasion (yes/no), and NLRc $(\leq 5.29 /$ / 5.29) were the independent risk factors for LN metastasis. Similarly, Japanese Gastric Cancer Association (JGCA) points that nonulcerated, well-differentiated and lesions diameter less than $2 \mathrm{~cm}$ are the indications for EMR/EDS approach in EGC patients[24]. Additionally, our study also found that the invasive depth of EGC(T stage) and vascular invasion were also associated with lymph node metastasis, Chu YN et al also found that submucosal invasion depth and LVI were the predictive factors for lymph node metastasis[25], hence, it is truely for the application of ultrasound gastroscope to identify the invasive depths in EGC patients, which could not only measured the lesion size, but also measured the invasive depths of EGC tumors[26]. In collaboration with previous studies, our research indicated that tumor size $\leq 1.9 \mathrm{~cm}$, ultrasound gastroscope presents the non-submucosa invasion and non-vascular invasion might be the indications for ESD/EMR in EGC patients.

There are several limitations in our study. Due to the retrospective nature of the study, some information(such as H.pylori infection, E.B virus infection et, al.) could not be collected; therefore, the analysable risk factors for those patients are still limited, and more data should be considered and collected. In addition, our findings should be verified by large-scale, multiple-center corhort. Moreover, our 
research retrospected from 2013 to 2018, which might be affected by the developments of practices and surgical expertise in different surgeons. Furthermore, adequate interventions should be applied to improve the EGC prognosis as patients have such poor prognostic factors.

\section{Conclusion}

The longest diameter of the tumor and total number of dissected LNs were independent prognostic factors for EGC patients. Additionally, the longest diameter of the tumor, tumor invasive depths, vascular invasion and NLRc were the independent risk factors for lymph node metastasis in EGC patients.

\section{Declarations}

\section{Ethics approval and consent to participate}

The research was supported by the Medical Ethical Committee of Xiangya Hospital, Central South University (No. 202004082).

\section{Consent for publication}

Not applicable.

\section{Availability of data and materials}

The raw data underlying this paper are available upon request to the corresponding author due to ethical restrictions.

\section{Competing interests}

The authors declare that they have no competing interests.

\section{Funding}

Not applicable.

\section{Authors' contributions}

Conceived and designed the experiments: Liao G

Analyzed the data: Qi J. Performed the experiments: Qi J, Zhu C, Liu S, Liu W, Cai G.

Wrote the paper: Qi J, Liao G. All authors read and approved the final manuscript.

\section{Acknowledgments}

Not applicable. 


\section{References}

1. Pizzi M, Saraggi D, Fassan M, Megraud F, Di Mario F, Rugge M. Secondary prevention of epidemic gastric cancer in the model of Helicobacter pylori-associated gastritis. Dig Dis. 2014;32(3):265-74.

2. Bray F, Ferlay J, Soerjomataram I, Siegel RL, Torre LA, Jemal A. Global cancer statistics 2018 : GLOBOCAN estimates of incidence and mortality worldwide for 36 cancers in 185 countries. CA Cancer J Clin. 2018;68(6):394-424.

3. Parkin DM. The global health burden of infection-associated cancers in the year 2002. Int J Cancer. 2006;118(12):3030-44.

4. Guggenheim DE, Shah MA. Gastric cancer epidemiology and risk factors. J Surg Oncol. 2013;107(3):230-6.

5. Yamamoto H, Watanabe Y, Maehata T, Morita R, Yoshida Y, Oikawa R. An updated review of gastric cancer in the next-generation sequencing era: insights from bench to bedside and vice versa. World $\mathrm{J}$ Gastroenterol. 2014;20(14):3927-37.

6. Verdecchia A, Francisci S, Brenner H, Gatta G, Micheli A, Mangone L, et al. Recent cancer survival in Europe: a 2000-02 period analysis of EUROCARE-4 data. Lancet Oncol. 2007;8(9):784-96.

7. Luo M, Li L. Clinical utility of miniprobe endoscopic ultrasonography for prediction of invasion depth of early gastric cancer: A meta-analysis of diagnostic test from PRISMA guideline. Med (Baltim). 2019;98(6):e14430.

8. McGuire S World Cancer Report 2014. Geneva, Switzerland: World Health Organization, International Agency for Research on Cancer, WHO Press, 2015. Adv Nutr. 2016; 7(2): 418-9.

9. Hu B, El Hajj N, Sittler S, Lammert N, Barnes R, Meloni-Ehrig A. Gastric cancer: Classification, histology and application of molecular pathology. J Gastrointest Oncol. 2012;3(3):251-61.

10. Bergquist JR, Leiting JL, Habermann EB, Cleary SP, Kendrick ML, Smoot RL, et al. Early-onset gastric cancer is a distinct disease with worrisome trends and oncogenic features. Surgery. 2019;166(4):547-55.

11. Qi J, Liu S, Liu W, Cai G, Liao G. Identification of UAP1L1 as tumor promotor in gastric cancer through regulation of CDK6. Aging. 2020;12(8):6904-27.

12. Ding J, Liao GQ, Liu HL, Liu S, Tang J. Meta-analysis of laparoscopy-assisted distal gastrectomy with D2 lymph node dissection for gastric cancer. J Surg Oncol. 2012;105(3):297-303.

13. Park DI, Yun JW, Park JH, Oh SJ, Kim HJ, Cho YK. HER-2/neu amplification is an independent prognostic factor in gastric cancer. Dig Dis Sci. 2006;51(8):1371-9.

14. Luo X, He Y, Tang H, Cao Y, Gao M, Liu B, et al. Effects of HER2 on the invasion and migration of gastric cancer. Am J Transl Res. 2019;11(12):7604-13.

15. Grabsch H, Sivakumar S, Gray S, Gabbert HE, Müller W. HER2 expression in gastric cancer: Rare, heterogeneous and of no prognostic value - conclusions from 924 cases of two independent series. Cell Oncol. 2010;32(1-2):57-65. 
16. Janjigian YY, Werner D, Pauligk C, Steinmetz K, Kelsen DP, Jäger E, et al. Prognosis of metastatic gastric and gastroesophageal junction cancer by HER2 status: a European and USA International collaborative analysis. Ann Oncol. 2012;23(10):2656-62.

17. Li Y, Zhao Q, Fan LQ, Tan BB, Zhang ZD, Liu Y. Analysis of lymph node dissection range-related factors for early gastric cancer operation. Hepatogastroenterology. 2013;60(125):971-4.

18. Li H, Lu P, Lu Y, Liu C, Xu H, Wang S, et al. Predictive factors of lymph node metastasis in undifferentiated early gastric cancers and application of endoscopic mucosal resection. Surg Oncol. 2010;19(4):221-6.

19. Gotoda T, Yanagisawa A, Sasako M, Ono H, Nakanishi Y, Shimoda T, et al. Incidence of lymph node metastasis from early gastric cancer: estimation with a large number of cases at two large centers. Gastric Cancer. 2000;3(4):219-25.

20. Wu H, Wang L, He YL, Xu JB, Cai SR, Ma JP, et al. [Impact of clinicopathological features and extent of lymph node dissection on the prognosis in early gastric cancer patients]. Zhonghua Zhong Liu Za Zhi. 2013;35(7):509-13.

21. Kasakura Y, Fujii M, Mochizuki F, Asaki H, Kobayashi M. Gastrectomy with D2 lymph node dissection in gastric cancer: a retrospective study at a single institution. Int Surg. 2001;86(1):50-6.

22. Park SS, Park JM, Kim JH, Kim WB, Lee J, Kim SJ, et al. Prognostic factors for patients with nodenegative gastric cancer: Can extended lymph node dissection have a survival benefit? J Surg Oncol. 2006;94(1):16-20.

23. Abdelfatah MM, Barakat M, Othman MO, Grimm IS, Uedo $\mathrm{N}$. The incidence of lymph node metastasis in submucosal early gastric cancer according to the expanded criteria: a systematic review. Surg Endosc. 2019;33(1):26-32.

24. Ono H, Yao K, Fujishiro M, Oda I, Nimura S, Yahagi N, et al. Guidelines for endoscopic submucosal dissection and endoscopic mucosal resection for early gastric cancer. Dig Endosc. 2016;28(1):3-15.

25. Chu YN, Yu YN, Jing X, Mao T, Chen YQ, Zhou XB, et al. Feasibility of endoscopic treatment and predictors of lymph node metastasis in early gastric cancer. World $\mathrm{J}$ Gastroenterol. 2019;25(35):5344-55.

26. Zhao B, Zhang J, Zhang J, Luo R, Wang Z, Xu H, Huang B. Risk Factors Associated with Lymph Node Metastasis for Early Gastric Cancer Patients Who Underwent Non-curative Endoscopic Resection: a Systematic Review and Meta-analysis. J Gastrointest Surg. 2019;23(7):1318-28.

\section{Figures}




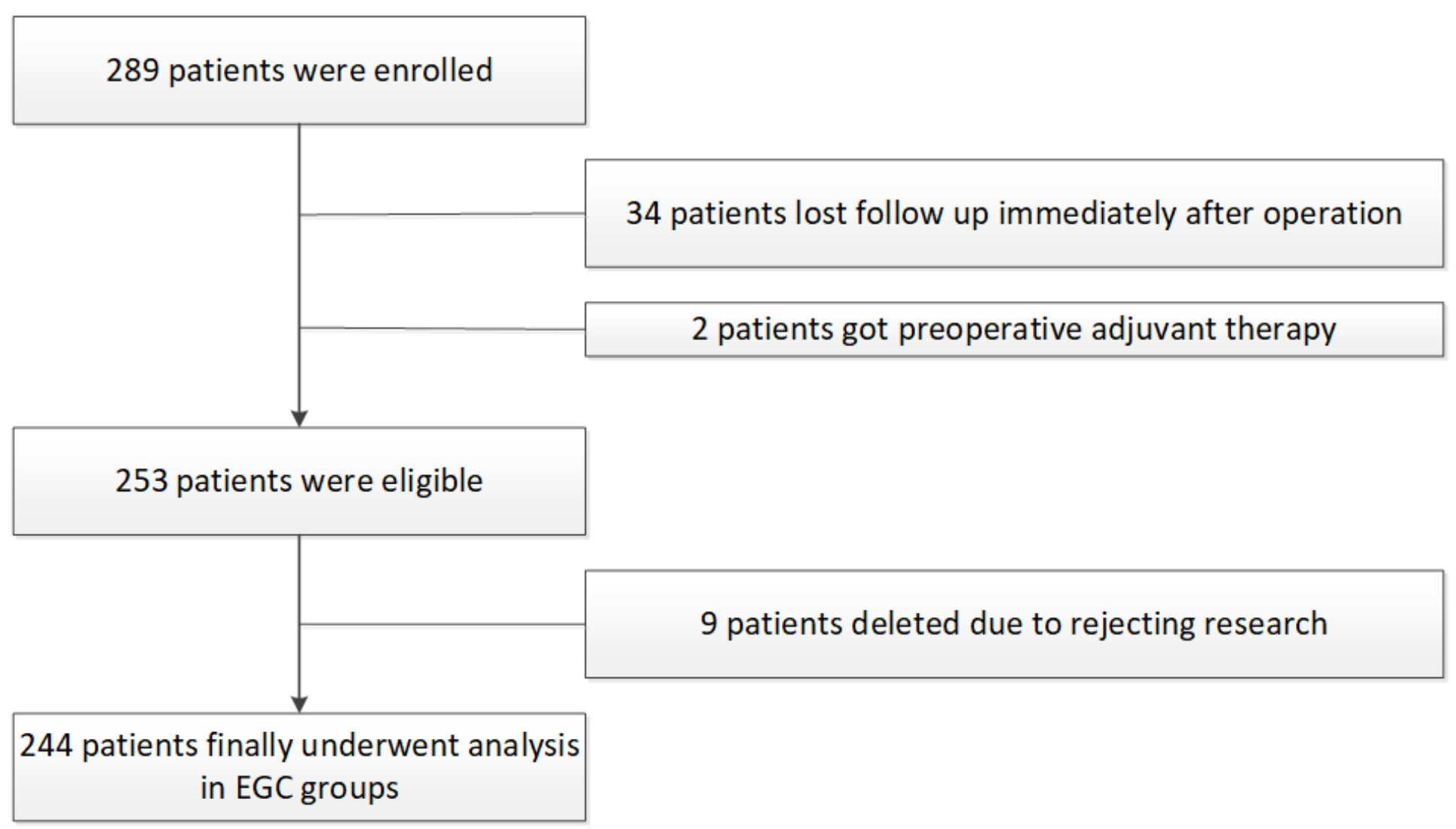

Figure 1

The study profile of patients enrolled in our study. 
a

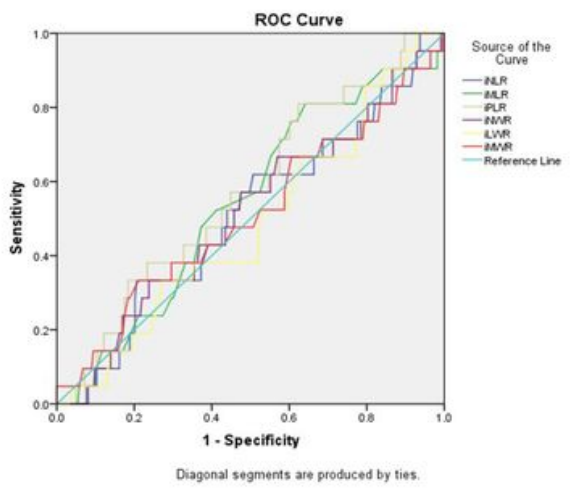

d

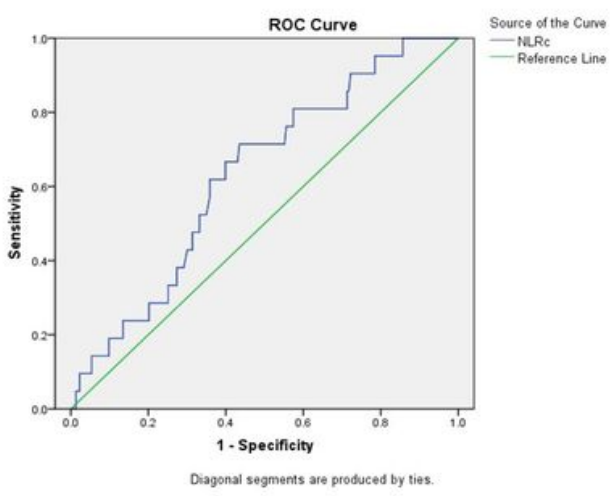

g

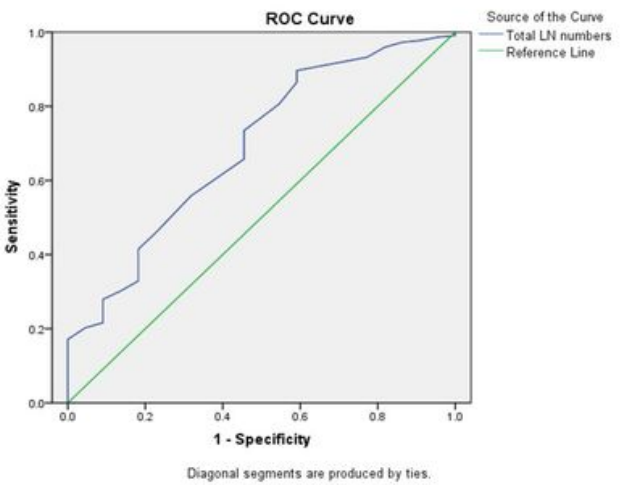

b

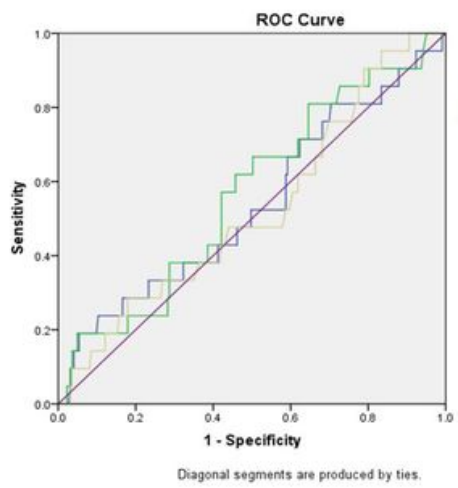

Source of the
Curve

二足A R

e

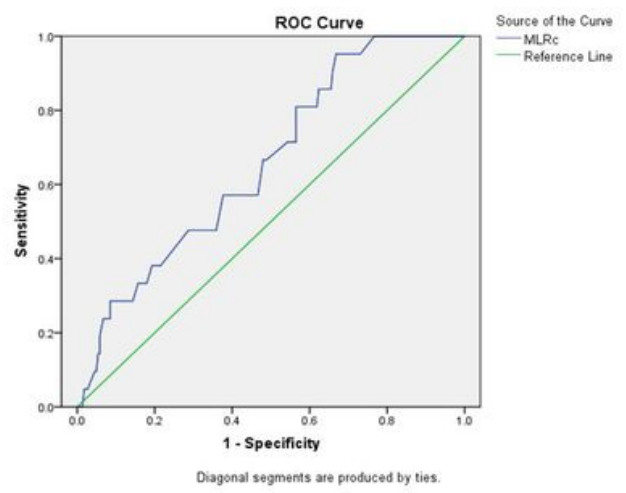

$\mathrm{h}$

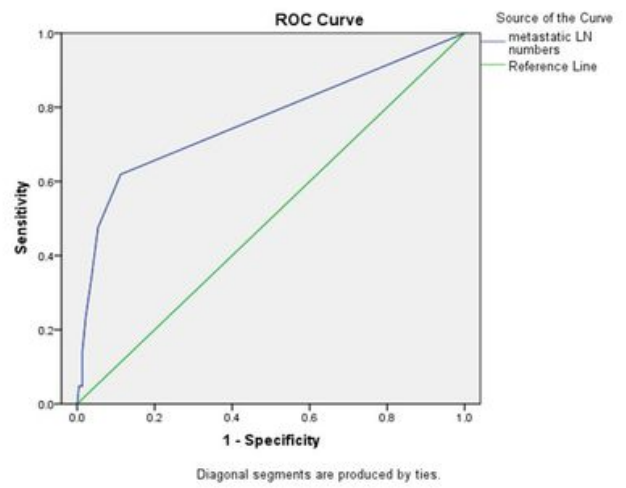

C
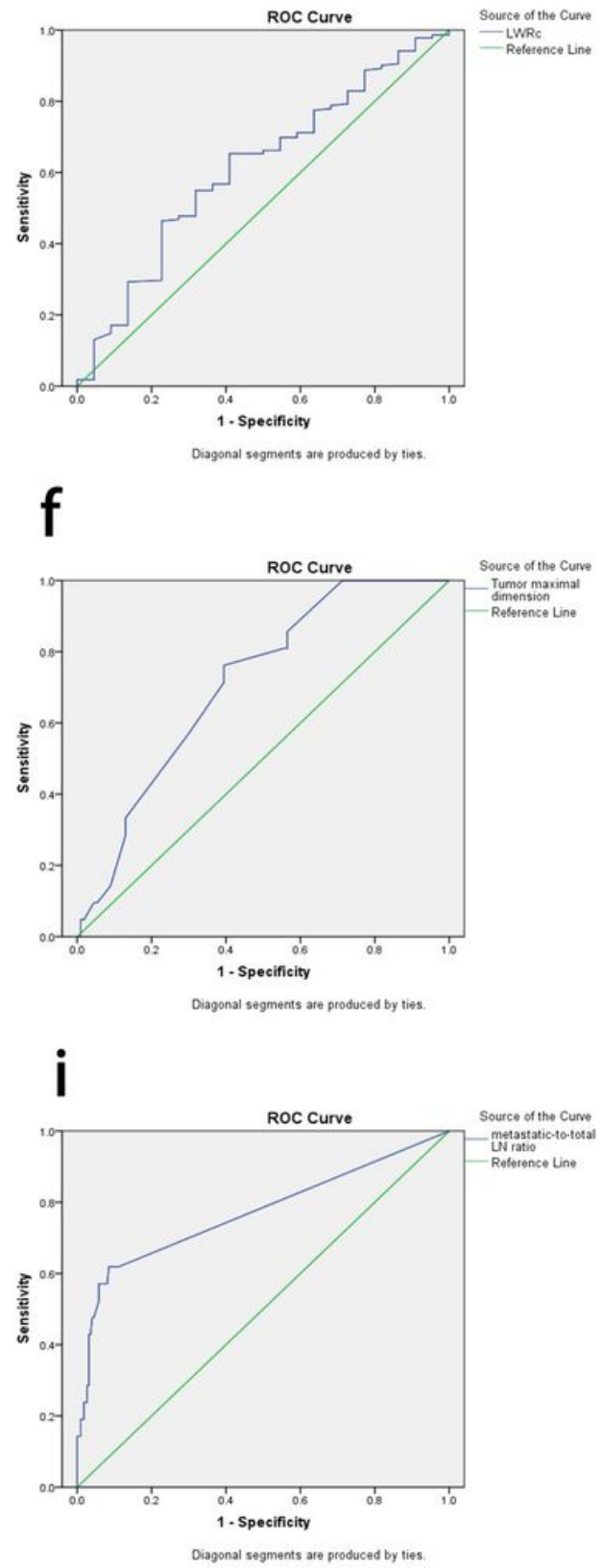

Figure 2

Receiver operating characteristic (ROC) curves for predicting disease-free survival among 244 early gastric cancer patients. (a) Preoperative parameters of routine blood analysis showed no significant prediction of DFS; (b) partial postoperative parameters (including PLRc, NWRc, and MWRc) of routine blood analysis showed no significant prediction of DFS; (c-i) in addition, ROC curves of LWRc, NLRc, MLRc, the longest diameter of the tumor, total number of dissected lymph nodes, number of metastatic lymph nodes, and ratio of metastatic-to-total dissected lymph nodes were used to predict disease-free survival. 

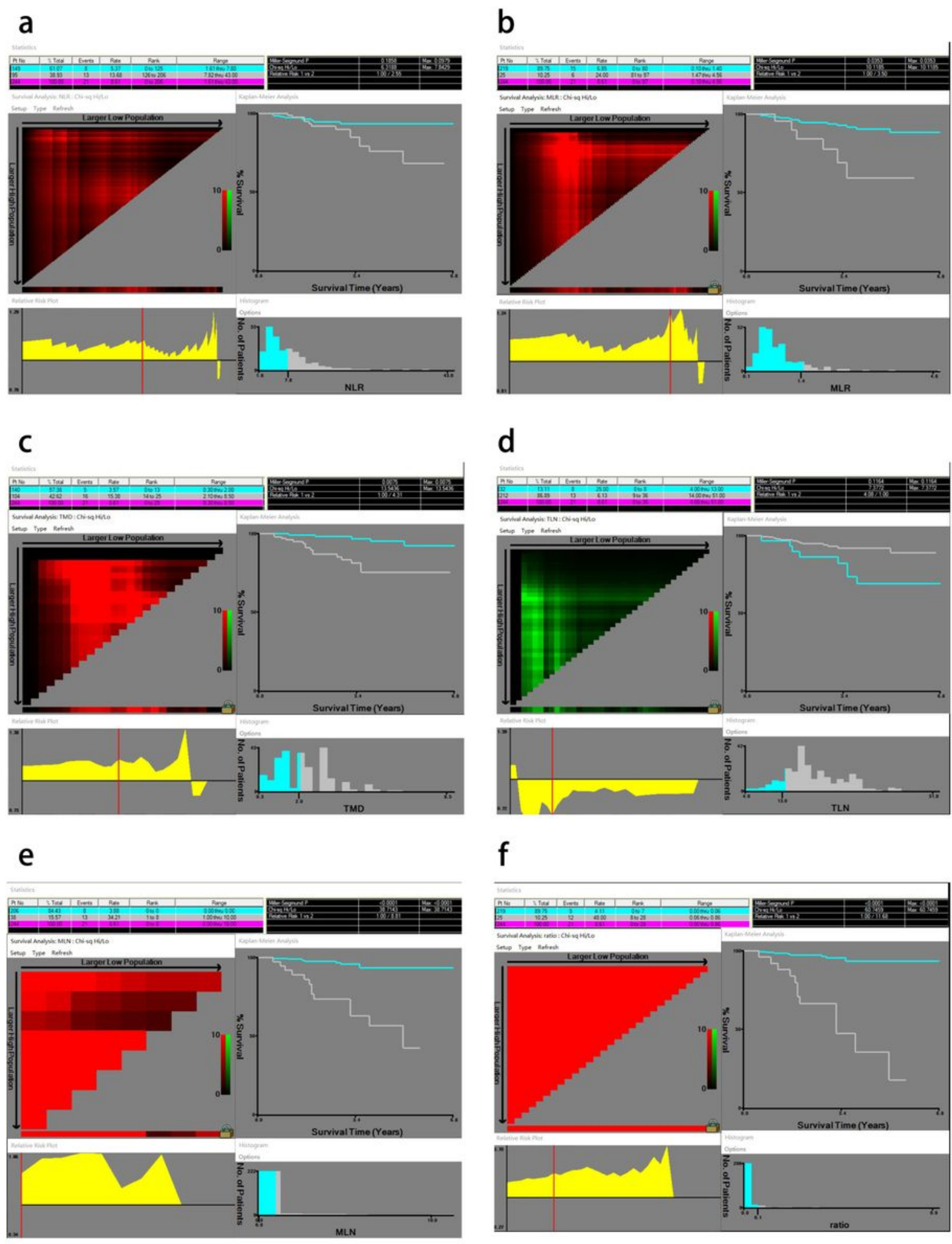

\section{Figure 3}

X-tile analysis of prognosis based on integral optic density (IOD). X-tile plots showing DFS among 244 EGC patients. (a-f) The data are stratified by NLRc, MLRc, the longest diameter of the tumor, total number of dissected lymph nodes, number of metastatic lymph nodes, and ratio of metastatic-to-total dissected lymph node. 
a
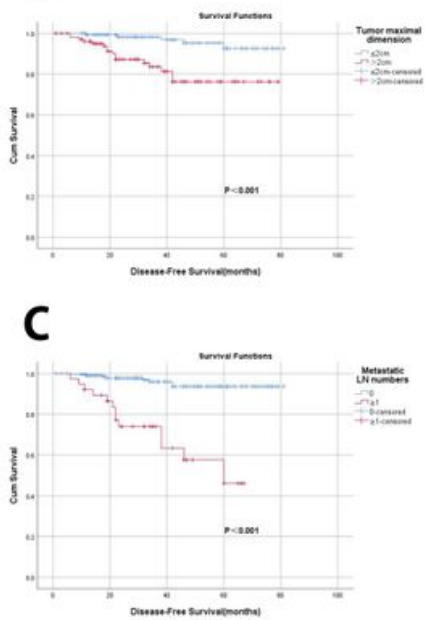

e

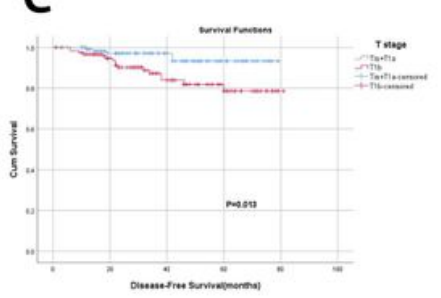

g

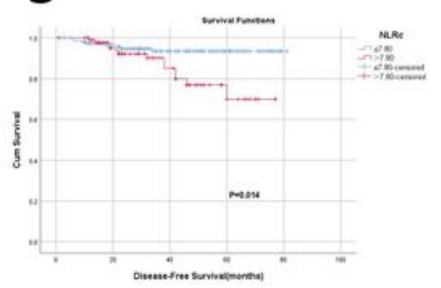

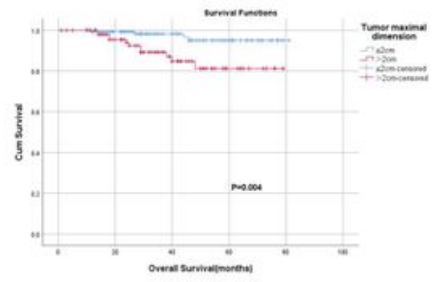
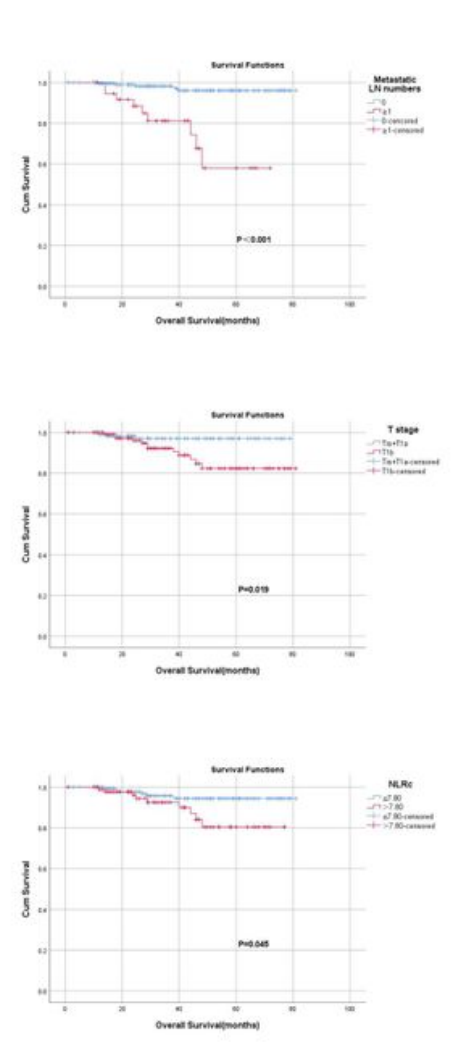

b
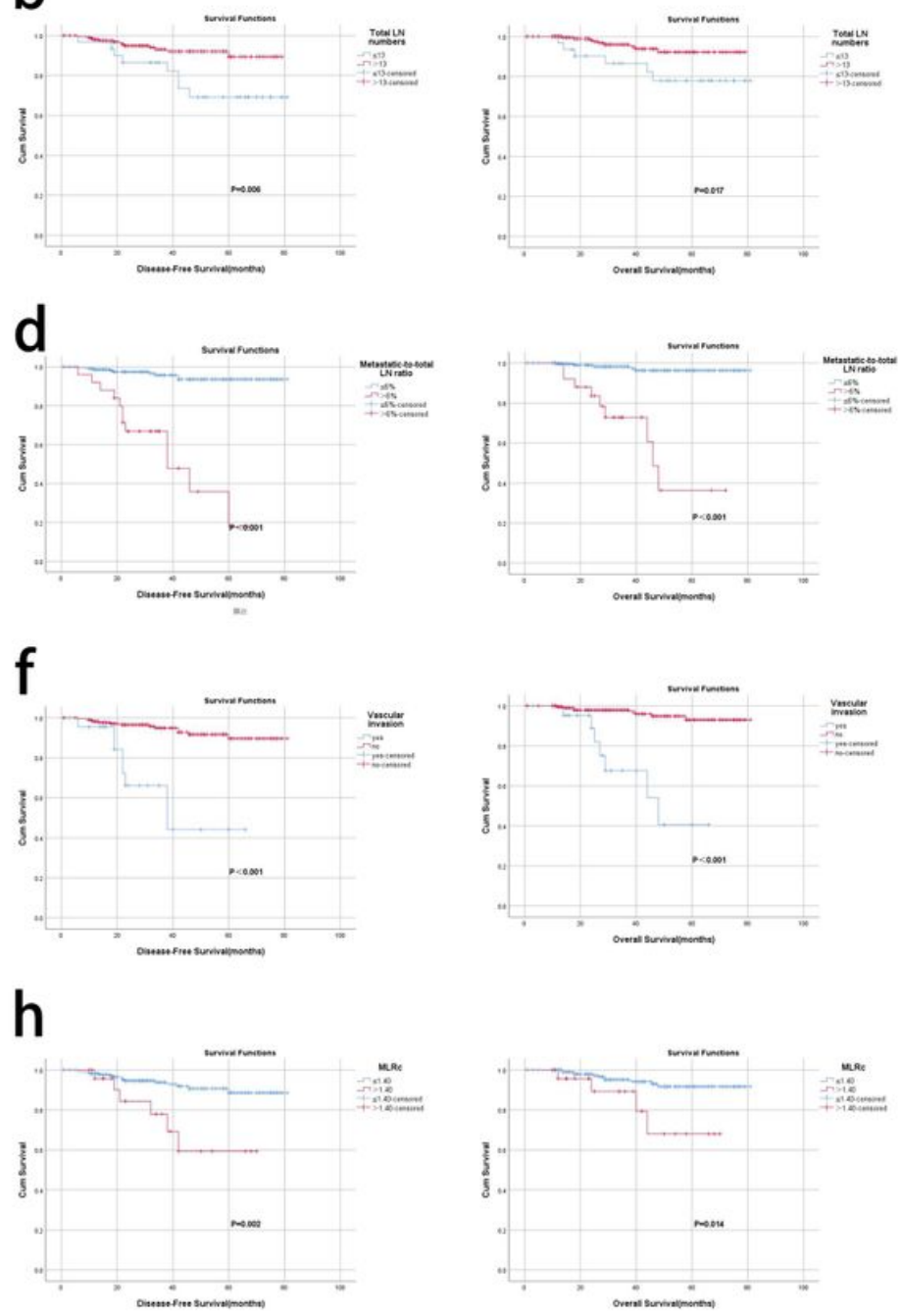

Figure 4

Disease-free survival and overall survival for 244 early gastric cancer patients. The data are stratified (a-i) by the longest diameter of the tumor ( $\leq 2 \mathrm{~cm}$ vs $>2 \mathrm{~cm}$ ), total number of dissected lymph nodes ( $\leq 13$ vs $>13$ ), number of metastatic lymph nodes ( $0 \mathrm{vs} \geq 1$ ), ratio of metastatic-to-total dissected lymph nodes ( $<6 \%$ vs $\geq 6 \%$ ), T stage (Tis+T1a vs T1b), vascular invasion (yes vs no), NLRc ( $\leq 7.80$ vs $>7.80$ ), and MLRc $(\leq 1.40$ vs $>1.40)$ based on the univariate analyses. 
a

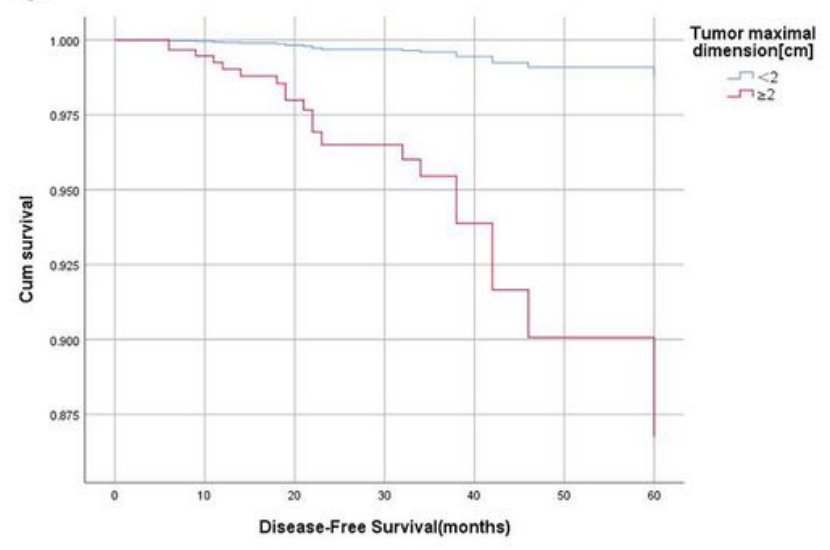

C

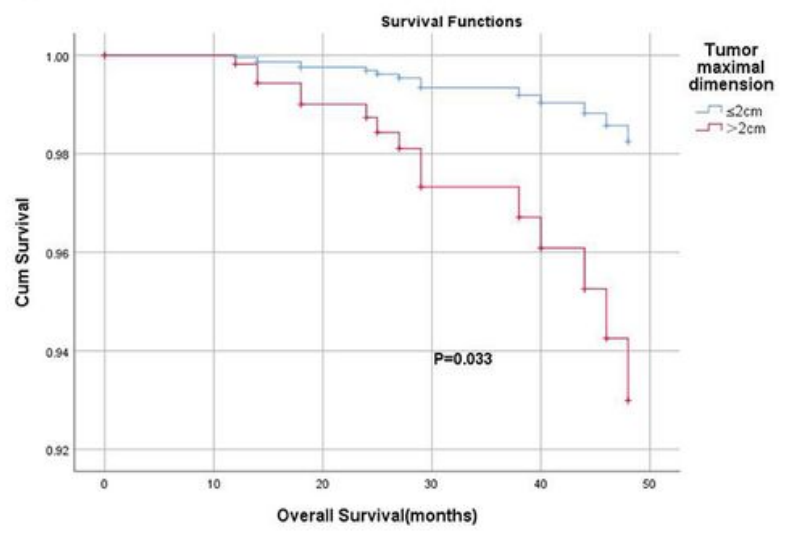

b

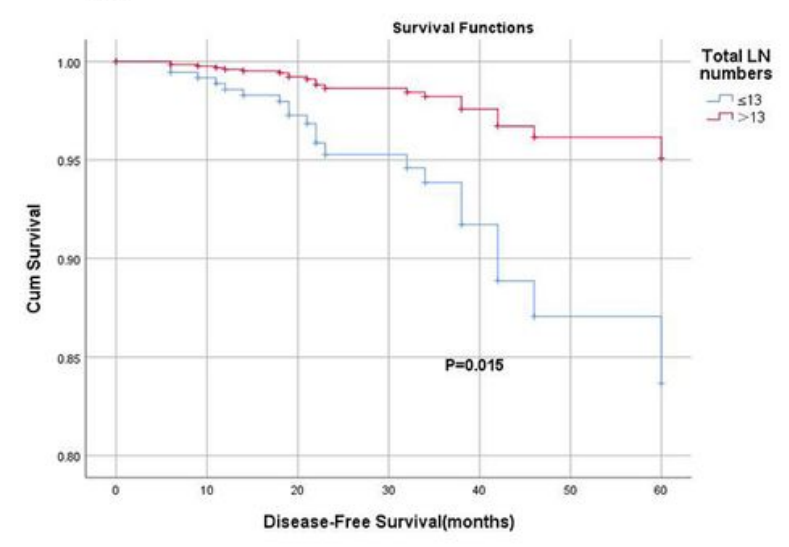

Figure 5

Disease-free survival and overall survival for 244 early gastric cancer patients according to multivariate analysis. The data are stratified by (a) the longest diameter of the tumor $(\leq 2 \mathrm{~cm}$ vs $>2 \mathrm{~cm}$ ) for recurrence/metastasis in EGC patients; (b)total number of dissected lymph nodes ( $\leq 13 \mathrm{vs}>13$ ) for recurrence/metastasis in EGC patients; (c)the longest diameter of the tumor ( $\leq 2 \mathrm{~cm}$ vs $>2 \mathrm{~cm}$ ) for overall survival in EGC patients.

\section{Supplementary Files}

This is a list of supplementary files associated with this preprint. Click to download.

- tableS1.docx

- FigureS1.tif 